\title{
POLÍTICA MONETARIA NO CONVENCIONAL EN EE.UU Y COMPORTAMIENTO DE LOS MERCADOS EMERGENTES EN AMÉRICA LATINA
}

\author{
NON-CONVENTIONAL MONETARY POLICY IN THE US AND BEHAVIOR OF \\ EMERGING MARKETS IN LATIN AMERICA
}

\section{POLÍTICA MONETÁRIA NÃO CONVENCIONAL NOS EUA E COMPORTAMENTO DE MERCADOS EMERGENTES NA AMÉRICA LATINA}

GIL-LEÓN_José Mauricio, TOCA-TOCA_Julián Santiago

\begin{abstract}
Magíster en Economía, Universidad Pedagógica y Tecnológica de Colombia. Profesor de Planta, Escuela de Economía, Universidad Pedagógica y Tecnológica de Colombia. E-mail: josemauricio.gil@uptc.edu.co, Colombia.
\end{abstract}

Magíster en Economía, Universidad Pedagógica y Tecnológica de Colombia. Profesor, Facultad de Negocios Internacionales, Universidad Santo Tomás de Aquino - Villavicencio. E-mail: juliansantiagot@gmail.com, Colombia.

Recibido: 14 de diciembre de 2019

Aprobado: 14 de abril de 2020

DOI: https://doi.org/10.22267/rtend.202101.126

\section{RESUMEN}

Este artículo pretende determinar la incidencia de la política monetaria no convencional de Estados Unidos implementada desde la crisis económica de 2008 sobre cinco mercados emergentes de América Latina. Por lo tanto, se identificó un canal de transmisión de los choques externos, los cuales afectaron en primera medida el rendimiento de los índices bursátiles, y luego se generó el traspaso a 
las variaciones de las tasas de cambio debido a la expansión de la entrada de flujos capital extranjero a los países en forma de inversión de cartera. También se evidenció, a través de la estimación de modelos de datos panel, la respuesta positiva de los flujos de capital de inversión de cartera a las expansiones de la hoja de balance en cada una de las fases de flexibilidad cuantitativa.

Palabras clave: política monetaria, relajación cuantitativa, índices bursátiles, flujos de capital. JEL: F32, F36, F44, F49, G01, G11, G19

\section{ABSTRACT}

This paper intends to determine the incidence of the unconventional monetary policy of the United States implemented since the economic crisis of 2008 on five emerging markets in Latin America. Therefore, a transmission channel for external shocks was identified, which first affected the return of the stock indices, and then the pass-through to changes in exchange rates was generated due to the expansion in the of foreign capital inflows to countries in form of portfolio investment. The positive response of portfolio investment capital flows to the balance sheet expansions in each of the phases of quantitative easing was also evidenced through the estimation of panel data models.

Keywords: monetary policy, quantitative easing, stock market indices, capital flows.

JEL: F32, F36, F44, F49, G01, G11, G19

\section{RESUMO}

Este artigo tem como objetivo determinar o impacto da política monetária não convencional dos Estados Unidos implementada desde a crise econômica de 2008 em cinco mercados emergentes da América Latina. Portanto, foi identificado um canal de transmissão de choques externos, que afetou primeiro o retorno dos índices de estoque e, em seguida, foi gerado o repasse às mudanças nas taxas de câmbio devido à expansão do fluxo de entrada de capitais estrangeiros para os países na forma de investimento de portfólio. A resposta positiva dos fluxos de capital de investimento do portfólio às expansões do balanço em cada uma das fases da flexibilidade quantitativa também foi evidenciada através da estimativa de modelos de dados em painel. 
Palavras-chave: política monetária, relaxamento quantitativo, índices de ações, fluxos de capital JEL: F32, F36, F44, F49, G01, G11, G19

\section{INTRODUCCIÓN}

A finales de 2008, la crisis económica generaba repercusiones negativas en la economía con la contracción del consumo y la reducción del crédito, llevando a la caída en el rendimiento de los bonos, acciones y derivados financieros. Importantes firmas hipotecarias y de inversión debieron recurrir al uso de dinero público para mantenerse a flote, pero esto acentuó el problema de liquidez, lo cual llevó a la Reserva Federal de Estados Unidos (Fed) a realizar la expansión monetaria (Martin, García y Arilla, 2010). A partir de ese momento la autoridad monetaria de Estados Unidos recurrió a la política monetaria no convencional (PMNC) para reactivar la economía y, así, dar un aliento a los mercados financieros (Caruana, 2011).

En el marco de la PMNC se implementó The Quantitative Easing (QE), definida como la adquisición por parte de la Reserva Federal de deuda pública, bonos privados y títulos relacionados con las causas de la crisis, llevando así al incremento de la base monetaria por medio de las reservas bancarias, comprando los activos mediante operaciones de mercado abierto (Rubai, 2013). De este modo, la relajación cuantitativa se realizó en cuatro fases: la primera (QE1) ${ }^{1}$ ocurrió a finales de 2008, con la cual la Fed negoció USD 600.000.000 millones, discriminados en: USD 100.000 millones en obligaciones de GSE ${ }^{2}$ y USD 500.000 millones en $\mathrm{MBS}^{3}$. Sus tenedores eran Fannie Mae, Freddie Mac y Federal Home. Después se realizaría otra compra por USD 300.000 millones de bonos americanos para un total de USD 1.750 millones, tal como lo había informado el presidente de la Fed (Morales, 2014, p.23).

La segunda fase de QE2 fue realizada en agosto de 2010 para la compra de USD 600.000 millones en Letras del Tesoro de Estados Unidos, con el propósito de rebajar el tipo de interés de los títulos del Tesoro y, de esa manera, reactivar la inflación vía aumento de consumo (Morales, 2014, p. 24). Seguido a esto, la Fed usó otras herramientas de política, las cuales consistieron en el intercambio de

\footnotetext{
${ }^{1}$ Relajación cuantitativa (Quantitative easing).

${ }^{2}$ GSE-Títulos de empresas patrocinadas por Estados Unidos- goverment-sponsored enterprises

${ }^{3}$ MBS- Instrumentos financieros que respaldan préstamos hipotecarios- mortgage backed securities.
} 
los títulos de deuda, invirtiendo los de corto plazo por largo plazo. La operación fue de la siguiente manera: se vendían los títulos con vencimiento de corto plazo y se compraban bonos con plazos entre seis meses y treinta años. A la vez, se mantenía el proceso de compra de activos como los MortgageBacked Securities (MBS) para influir en los tipos de interés de largo plazo, buscando mantener la misma base monetaria, pero entregando más liquidez a la economía, a lo que también se denominó operación Twist.

En la tercera fase de relajación cuantitativa (QE3) el objetivo de esta compra por USD 400.000 fue en noviembre de 2012, pero se terminaron comprando 45.000 millones de lo previsto en Letras del Tesoro. Esta fase posee la particularidad que aumentó la base monetaria, debido a que no se estaba haciendo con el método tradicional de operaciones inversas de los plazos de los activos, a diferencia de las demás fases en la QE3 la Fed sí aumentó el dinero en circulación. El dinero del banco central se mide con la base monetaria (BM), conformada por las reservas bancarias de las instituciones de depósitos y el efectivo en circulación dentro de Estados Unidos.

Al mismo tiempo, las economías emergentes (EE), para atenuar los choques externos han venido empleando ciertas medidas recomendadas por el Fondo Monetario Internacional (FMI), las cuales se basan en el acceso a líneas de crédito flexible (FCL, por sus siglas en inglés) que beneficiaron a países como Colombia, México y Polonia, por un total de USD 77.000 millones de dólares. En la mayoría de las EE de América Latina (AL) se diseñaron programas para controlar los ataques especulativos y, por esta razón, para tener una visión más acertada de cómo estos choques afectaron las EE de la región.

En este artículo se busca identificar los efectos de las medidas no convencionales sobre los mercados emergentes de AL, según JP Morgan, tales como: Brasil, Chile, Colombia, México y Perú (se excluye Argentina). Por lo tanto, el documento se divide en cuatro secciones, considerando a esta como la primera. En la segunda sección se realiza una revisión de estudios sobre los efectos de la PMNC en los mercados emergentes. En la tercera se describe la metodología usada para los análisis. En la cuarta se analizan los efectos de la PMNC sobre los mercados de activos financieros, y también se aborda la medición de las implicaciones sobre los flujos de capital. Finalmente, se formulan algunas conclusiones. 


\section{REFERENTES TEÓRICOS}

Dentro de los estudios más relevantes se encuentra el documento de Gilchrist (2014), en el cual se cuantificó el impacto de los choques de la PMNC de Estados Unidos, y el grado de transmisión de esas medidas sobre el rendimiento de los bonos extranjeros en relación a los bonos a dos y diez años de Estados Unidos. Gilchrist (2014) evidencia cómo la diferencia entre el redimiento de los bonos americanos de 10 y 2 años de vencimiento se reduce, por lo que se tiende a aplanar la curva de rendimientos, debido a los efectos de las medidas no convencionales; además, destaca que cambios en las tasas de interés de los bonos del Tesoro llevan a que los rendimientos de los bonos extranjeros aumenten en medidas convencionales y se reduzcan con el uso de medidas no convencionales.

Es así como Bowman et al. (2015) y Tillmann (2016) calculan cómo la PMNC de EE. UU afectó a los mercados emergentes, señalando que las medidas incidieron sobre los rendimientos de los bonos soberanos, las tasas de cambio se modificaron, las fluctuaciones de los precios de las acciones y los flujos de capital (Chen et al., 2014). En el caso de la mayor entrada de flujos de capital, estos se dirigieron especialmente hacia inversiones de cartera (Ahmed y Zlate, 2014). Las estimaciones muestran que el tamaño de los efectos está sujeto a las características de cada uno de los países emergentes (Chen et al., 2014 y Bowman, et al., 2015), y además fueron débiles (Ahmed y Zlate, 2014). Sin embargo, se determina que los efectos de los choques de tasas de interés son siempre más fuertes que los asociados a las medidas no convencionales (Bowman et al, 2015).

Dentro de los trabajos que han explicado el papel de la PMNC en los mercados emergentes de América Latina está el de Otero (2015), en el cual se realizó un estudio sobre el comportamiento de las economías de Chile, Brasil, Colombia, Perú y México, y cómo estas han reaccionado a las medidas de PMNC bajo regímenes de inflación objetivo, mostrando que la economía con mayor agilidad en la respuesta a un choque externo es la mexicana, y la más lenta en responder al choque es la colombiana. Se evidencia que en México las variaciones en la tasa de cambio tienen mayor efecto sobre la apreciación de la moneda, pero contrae la actividad productiva, mientras que en Brasil, Chile y Perú los efectos operan de manera opuesta (Otero, 2015).

Con la implementación de las medidas no convencionales, las economías emergentes desde 2010 se habían convertido en los mercados con mejor dinámica en su comercio exterior, con una tasa de 
crecimiento estable, y una mayor entrada de flujos de capital financiero (Orgaz, Molina, y Carrasco, 2011). De lo anterior se expresa que los países con mayores variaciones en sus flujos de capital son los más expuestos a las crisis, que en este caso son Brasil, Colombia y México. Igualmente, para el caso de Perú y Chile, la autoridad monetaria de cada país mantuvo una conducta de disminución de la tasa de interés lo más cercana al cero por ciento (Orgaz, Molina, y Carrasco, 2011).

Así mismo, una de las razones por las que aumentó los flujos de capital hacia AL desde el 2010 hasta el 2014, fue la baja probabilidad de que la autoridad monetaria de cada país en AL disminuyera la tasa de interés de una manera inesperada, garantizando un mayor margen de retorno sobre los flujos (CEPAL, 2016). Esta tendencia acompañada del alto precio de los commodities favoreció a la región cerca de cuatro años. La recuperación de las economías desarrolladas llevó a la contracción de los flujos de capital en la región, los cuales disminuyeron un 8\% en 2016 frente al 2015, lo que implicó una pérdida del 17\% de los flujos de capital en relación a 2011 (CEPAL, 2016).

Con respecto de las vulnerabilidades de las economías de AL, para Calvo y Perry (2015) los riesgos financieros causados por la contracción de la hoja de balance de la Fed han generado que los niveles de liquidez en los mercados financieros disminuyeran, aumentando la volatilidad de los rendimientos de los activos y la salida de capitales. En ese sentido, la normalización de la política monetaria implicó una salida de flujos de cartera, especialmente cuando las tasas de interés comiencen a subir en las economías desarrolladas, aunque es de aclarar que las repercusiones son relativamente pequeñas en términos de impacto económico (Dahlhaus y Vasishtha, 2014). Otro escenario de atención y control por parte de las EE, es la recuperación del dólar, frente a la mayoría de las monedas de la región, lo que genera el encarecimiento de la deuda externa de cada país, restringiendo las opciones de financiamiento internacional (Calvo y Perry, 2015).

\section{METODOLOGÍA}

En este trabajo se implementan dos tipos de análisis: 1) la estimación de la relación entre la volatilidad de los rendimientos de los activos financieros de las EE y la política monetaria implmentada en EE.UU después de la crisis de 2008; y 2) el análisis de la incidencia de la flexibilidad cuantitativa sobre los flujos de capital hacia AL. 


\section{Relación entre la PMNC y la volatilidad del rendimiento de los activos financieros}

Se estiman modelos GARCH para determinar la volatilidad diaria de los índices bursátiles y las tasas de cambio desde 2007 hasta 2017. Este periodo de tiempo está caracterizado por ser el punto de partida de la PMNC de Estados Unidos y el ciclo de auge de los commodities en la región de AL.

$$
\sigma_{t}^{2}=\omega+\alpha \varepsilon_{t-1}^{2}+\delta \sigma_{t-1}^{2}
$$

Los modelos GARCH poseen una estructura de la varianza condicional ${ }^{\left(\sigma_{t}^{2}\right)}$ conformada por la media $(\omega)$, la varianza del día anterior $\left(\sigma_{t-1}^{2}\right)$ y la información acerca de volatilidad en el periodo anterior $\left(\varepsilon_{t-1}^{2}\right)$. Esta relación permite que se capture de manera más completa los ciclos de volatilidad (Monsegny y Cuervo, 2008). Por esta razón el modelo empleado es la versión clásica del Garch.

En la ecuación 2 se específica el modelo de volatilidades del Índice Bursátil (IB) y de la Tasa de Cambio (TC):

$$
\operatorname{garhc}_{t}=\beta_{0}+\beta_{1} \Delta b m_{t}+\mu_{t}
$$

Cada $\beta_{1}$ representa el comportamiento de la volatilidad del índice bursátil diario de las cinco economías emergentes (BOVESPA, IPSA, IGBC, IPC, IGBVL) y de las tasas de cambio diarias por país (Brasil, Chile, Colombia, México y Perú). Esto en función del índice de la base monetaria $\left(\Delta b m_{t}\right)$ que representa la variación de la base monetaria de Estados Unidos diariamente desde el 2007 hasta el 2017. La $\Delta b m_{t}$ se determina tomando como base el tamaño de la hoja de balance en noviembre de 2008, y a partir de allí se determina la proporción del incremento.

Después de analizar la volatilidad de los IB y del TC por país se diseña modelos VAR (Vectores AutorRgresivos) bivariados con datos diarios entre 2007 y 2017 para cada país, con el propósito de definir cómo es la dinámica de la relación entre estas dos variables, donde la dirección de la relación se estimó a través del test de causalidad de Granger. Se encontró que el principal canal de traspaso de choques externos es de la siguiente manera: una perturbación externa, en primera medida, impacta en los mercados financieros y luego estas perturbaciones inciden en el mercado cambiario. 
El uso de un modelo VAR permite definir de manera más precisa cómo son los procesos de correlación y transmisión de los efectos entre las variables, autores como Ramirez y Rodriguez (2013) destacan que el modelo VAR logra analizar las relaciones contemporáneas y los efectos dinámicos. Esto llevó al cálculo de las matrices contemporáneas y los efectos dinámicos de choques de la variación en los índices bursátiles sobre las tasas de cambio nominal. La metodología es usada puesto que los modelos VAR permiten captar de una mejor manera los efectos dinámicos, ya que al tener la tasa de cambio nominal y el índice bursátil diarios se logra estimar el canal de los efectos de la PMNC. Es importante resaltar que el efecto de contagio en el sector financiero es mucho más rápido, lo que permitió establecer un canal de traspaso que después de la estimación del VAR y el test de causalidad se logró evidenciar.

\section{Identificación de la relación entre los flujos de capital hacía EE y la PMNC}

Se analiza el comportamiento de los flujos de capital extranjero hacia AL en función de las medidas no convencionales. Los efectos de las medidas no convencionales sobre los flujos de capital se miden a través de un modelo de datos panel, cuya estructura brinda información precisa sobre las especificidades a lo largo del periodo de análisis, y por tanto se controla la heterogeneidad no observada, la cual no se puede captar por medio de series temporales o de corte transversal (Perazzi y Merli, 2013). En este punto los modelos de datos panel permiten estimar esos efectos no observables, que usualmente son efectos individuales específicos y efectos temporales (Wooldridge, 2010).

El modelo de datos panel estimado tiene dominancia en series de tiempo, y define una relación positiva con el comportamiento de los flujos de capital. Toro-Córdoba et al. (2012) muestran que los flujos de capital en la región y desde la región crecieron considerable antes de la crisis, como resultado de los procesos de globalización financiera. El comportamiento de los flujos de capital está relacionado con la naturaleza del mismo ya que, al ser una crisis de origen financiero, el flujo de inversión de cartera mostró alto nivel de volatilidad. No obstante, la crisis también tuvo efectos sobre las restricciones de liquidez y en la reducción del financiamiento externo para AL, lo que generó repercusiones sobre el comercio internacional, la producción y el nivel de empleo.

Los flujos de inversión de cartera son uno de los determinantes claves para entender el comportamiento de los flujos en AL, y así mismo, son sensibles a perturbaciones externas, dado que 
el tipo de activos financieros que se usan poseen un alto nivel de volatilidad en el corto plazo, y especialmente tienen una relación directa con las decisiones de rentabilidad. Es importante destacar que dentro del periodo analizado el flujo de inversión extrajera directa aumentó, en especial desde el 2010-2014, debido a que gran parte de este recurso se concentró en la industria de extracción, pero un mayor ingreso de esta clase de capital no implicó mejores condiciones para las economías.

Las economías emergentes tiene como reto el de atraer mayores niveles de inversión que contribuyan al desarrollo sostenible y al cambio estructural de la región (CEPAL, 2018). Este efecto lleva a que la inversión de cartera concentre un alto nivel de volatilidad, en función a esto, los activos de cartera negociados dependen en gran medida de las condiciones financieras de la economía residente, y la manera en cómo la economía asimila los choques externos, efecto que le permite a la inversión de cartera reaccionar en forma más volátil ante cada una de las medidas no convencionales. Analizar la incidencia de las medidas no convencionales sobre la inversión de cartera es primordial con el fin de establecer cómo el comportamiento de este flujo es determinante de la estabilidad macroeconómica. La ecuación a estimar es:

$$
\text { flujos_capital }_{i t}=\gamma_{0}+\gamma_{1} \Delta b m_{i t}+X_{i t} \delta+\mu_{i t}
$$

La ecuación (3) permite estimar la incidencia de las variaciones en la base monetaria de EE.UU sobre los flujos de capital extranjero de cartera mediante el valor de $Y_{1}$. El vector de variables de control $X_{i t}$ explica el comportamiento de los flujos de capital extranjero con el vector de coeficientes $\delta$. Se realiza una estimación para identificar el papel de la PMNC sobre la entrada de capital de extranjeros, y otra estimación sobre la salida de capital nacional hacia el extranjero. Las variables de control son: el crecimiento económico, la política monetaria del país, las operaciones con el sector externo respecto de la actividad económica, el crédito del sector financiero y la tasa de cambio.

La Tabla 1 muestra la especificación para la estimación por medio de un modelo de panel de la influencia de la PMNC sobre la entrada de flujos de capital de extranjeros: 


\section{Tabla 1}

Especificación de variables a utilizar en la primera estimación

\begin{tabular}{|c|c|c|}
\hline Tipo & Definición & Fuente \\
\hline $\begin{array}{l}\text { Inv. cartera en } \\
\text { pasivos (ICP) }\end{array}$ & $\begin{array}{l}\text { Es la inversión de no residentes en activos emitidos por } \\
\text { residentes como: acciones, bonos, pagarés y demás } \\
\text { instrumentos monetarios emitidos por residentes. }\end{array}$ & Bancos Centrales de cada país \\
\hline $\begin{array}{l}\text { Cambios de la } \\
\text { base monetaria }\end{array}$ & $\begin{array}{l}\text { Cambios de la proporción diaria en millones de dólares } \\
\text { de la base monetaria que incluye las reservas agregadas } \\
\text { totales de las instituciones de depósitos y la base } \\
\text { monetaria, serie entre el } 2007 \text { y } 2017 \text {. }\end{array}$ & $\begin{array}{l}\text { Reserva Federal del Banco de } \\
\text { St. Luis (2018) }\end{array}$ \\
\hline $\begin{array}{l}\text { Crecimiento } \\
\text { Económico }\end{array}$ & Variación porcentual del PIB a precios constante & Cepalstat (2018) \\
\hline $\begin{array}{l}\text { Tasa de política } \\
\text { monetaria }\end{array}$ & $\begin{array}{l}\text { Instrumento de intervención de política monetaria } \\
\text { usado por la autoridad monetaria de cada país para } \\
\text { afectar la cantidad de dinero que circula en la } \\
\text { economía, consiste en modificar la tasa de interés que } \\
\text { cobra a las entidades financieras por los préstamos, o } \\
\text { la tasa de interés máxima que paga por recibir dinero } \\
\text { sobrante de las entidades comerciales }\end{array}$ & Bancos Centrales de cada país \\
\hline $\begin{array}{l}\text { Cuenta } \\
\text { Corriente } \\
\text { respecto del PIB }\end{array}$ & $\begin{array}{l}\text { Es la suma de las exportaciones netas de bienes, } \\
\text { servicios, ingresos netos y transferencias corrientes } \\
\text { netas, como porcentaje del PIB }\end{array}$ & $\begin{array}{l}\text { Fondo Monetario } \\
\text { Internacional (2018) }\end{array}$ \\
\hline $\begin{array}{l}\text { Crédito como } \\
\text { proporción del } \\
\text { PIB }\end{array}$ & $\begin{array}{l}\text { Son los recursos proporcionados al sector privado por } \\
\text { las entidades financieras, a través de préstamos, } \\
\text { compras de valores no condicionales y créditos } \\
\text { comerciales y otras cuentas por cobrar, que establecen } \\
\text { un reclamo de reembolso. Crédito de todo el sector } \\
\text { privado, al sector no financiero, como \% del PIB }\end{array}$ & $\begin{array}{l}\text { Fondo Monetario } \\
\text { Internacional (2018) }\end{array}$ \\
\hline
\end{tabular}


Es el precio de una unidad del dólar de Estados Unidos

Tasa de Cambio expresado en términos de la moneda nacional y corresponde al promedio de los tipos de cambio de compra y venta

Fuente: elaboración propia con datos de los autores.

En la Tabla 1 se presenta las variables contempladas para la estimación de los determinantes de la entrada de flujos de capital extranjero, y algunas están como proporción del PIB, lo que permite evidenciar la participación de la variable con respecto de la actividad económica del país. El análisis econométrico se realizó con tres tipos de estimaciones: en primer lugar por medio de mínimos cuadrados ordinarios (MCO) para la consistencia de la especificación, luego se pasó al cálculo de efectos fijos (FE), y efectos aleatorios (RE), y por medio del test de Hausman (1978) se selecciona el estimador estadísticamente consistente, aunque efectos fijos tiene ventajas en el sentido que el efecto individual está correlacionado con las variables explicativas, lo que implica que el efecto individual es tratado separadamente del término de error. Labra y Torrecillas (2014) consideran que esto ayuda a reducir la sobreestimación del parámetro.

\section{Tabla 2}

Especificación de variables a utilizar en la segunda estimación

\begin{tabular}{lll}
\hline & \multicolumn{1}{c}{ Definición } & \multicolumn{1}{c}{ Fuente } \\
\hline Inv. Cartera & $\begin{array}{l}\text { Es la inversión de portafolio efectuada por residentes en títulos } \\
\text { emitidos por no residentes, comprende la adquisición de títulos } \\
\text { activos (ICA) }\end{array}$ & $\begin{array}{l}\text { Bancos Centrales } \\
\text { de participación en el capital y títulos de deuda. }\end{array}$
\end{tabular}

Cambios de la Cambios en la proporción diaria de la base monetaria que Reserva Federal base incluye las reservas agregadas totales de las instituciones de del Banco de St. monetaria depósitos y la base monetaria, serie entre el 2007 y $2017 . \quad$ Luis (2018) 
Fondo Monetario

Cuenta Es la suma de las exportaciones netas de bienes, servicios, Internacional

Corriente_\% $\mathrm{P}$ ingresos netos y transferencias corrientes netas, como (2018)

IB

porcentaje del PIB

Bancos Centrales

de cada país.

Son los recursos proporcionados al sector privado por las corporaciones financieras, como a través de préstamos, compras

Crédito_\%PI de valores no condicionales y créditos comerciales y otras B cuentas por cobrar, que establecen un reclamo de reembolso.

Para algunos países estas reclamaciones incluyen crédito a

Fondo Monetario

Internacional

empresas públicas, como porcentaje del PIB

Crecimiento Variación porcentual del PIB a precios corrientes en

Económico millones de dólares

Cepalstat (2018)

Fuente: elaboración propia con datos de los autores.

La Tabla 2 presenta la especificación usada para analizar el comportamiento de las salidas de capital de nacionales. Estos son los activos externos de cartera que se toman como proporción del PIB. De igual manera, se estimó la influencia de la base monetaria sobre este flujo por medio de un modelo de datos panel (en la Tabla 6 se evidencian los resultados).

\section{DISCUSIÓN}

\section{La PMNC y los rendimientos de activos financieros en EE de AL}

La agudización de la crisis ocurrió con la bancarrota de Lehman Brothers, permitiendo así que la incertidumbre y la volatilidad afectara la mayoría de los mercados financieros del mundo, y en especial a las EE, en un panorama donde la inyección de liquidez fue la mejor herramienta para reactivar la economía era inevitable que las economías emergentes no estuvieran expuestas al exceso de liquidez en los mercados, lo que implicó un aumento de la volatilidad de los activos financieros en la región.

En la Figura 1 se observa el crecimiento acelerado de la base monetaria después de 2008 hasta el 2013, que fue el nivel máximo de los incrementos. Existen consideraciones teóricas que señalan que el 
aumento drástico de la base monetaria por medio de la QE llevaría a niveles altos de inflación, lo que implicaba que los bancos e instituciones comerciales tenían un alto monto de reservas en la Fed, que en efecto aumentó la base monetaria, pues gran parte de dichas reservas eran usadas para líneas de créditos, pero si estás aumentaban drásticamente la inflación se iba a acelerar. Este escenario no ocurrió porque la Fed pagaba intereses por los recursos depositados, lo que garantizaba que el interés pagado para esas reservas era mayor que el retorno con respecto de otras opciones de inversión, lo que generó que las reservas quedaran estables (Ricketts, 2011). En la Figura 1 se observa la estabilidad de la base monetaria antes de noviembre de 2008 y posterior a 2014, es decir, durante el periodo de PMNC hubo expansión en la hoja de balance.

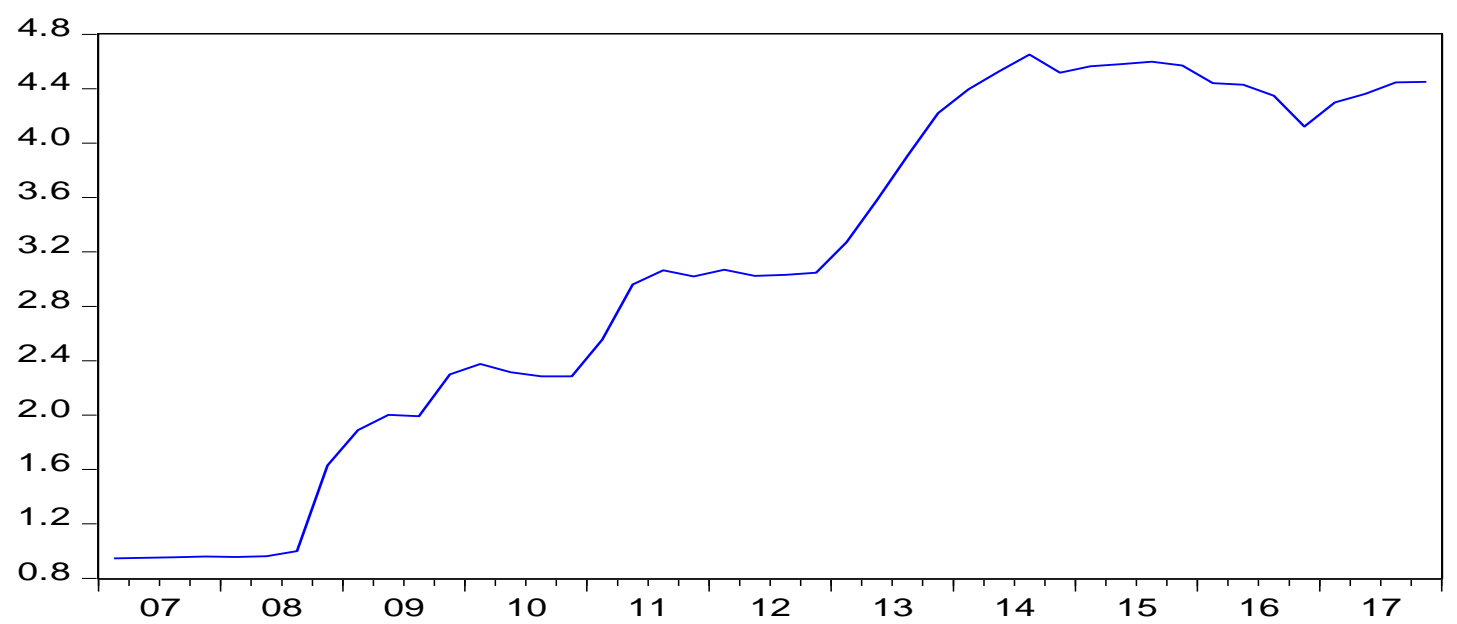

Figura 1. Índice de cambios de la base monetaria de Estados Unidos (noviembre 2008=1).

Fuente: elaboración propia con datos de la Fed (tomados en 2018).

La volatilidad estimada de los IB por país mediante el modelo GARCH se observa en la Figura 2. En esta se aprecia cómo la volatilidad del Bovespa no fue tan marcada como la del IGBC y el IPC. La economía brasileña posee varias ventajas con respecto de las demás en la región, ya que el nivel volatilidad de su indicador bursátil es bajo, pues es uno de los países que conforman el grupo de los BRICS. Con respecto al IPC, las condiciones de actividad comercial de México están relacionadas con Estados Unidos, así que le efecto contagio es alto.

Al estudiar las medidas la PMNC, se debe tener presente el contexto no solo de las economías desarrolladas, en este caso de Estados Unidos, sino también el ciclo que vivía AL después de la segunda medida de expansión monetaria, a partir de 2010, donde el nivel de volatilidad era bajo, que 
se explica por dos factores importantes en la AL, en primera medida, las bajas tasas de interés en países desarrollados, que ocasionaron un mayor flujo de capital en la región. Adicional, el auge en el precio de los commodities tomaba fuerza lo que mantenía a la región como una de las más rentables, efecto que se detuvo en el 2015, por aspectos como la normalización de la política monetaria en las economías avanzadas y la caída de precio del petróleo. Este efecto fue de mayor impacto en Chile, Colombia y Perú que para el 2015 mostraron episodios de volatilidad más altos (Ocampo, 2009).

En relación a lo expuesto por Ocampo (2009), el aumento de los flujos hacia la región, motivado por el precio del petróleo, gas, cobre, ferroníquel y oro llevaron al aumento de la inversión extranjera, y, por consiguiente, las bolsas de valores de la región dinamizaron su expansión (Rincón y Velasco, 2013). En el 2015 el ciclo llegó a su fin, con la contracción de los niveles de inversión, la disminución del valor de los títulos de deuda y la salida de capitales, ya que gran parte de las inversiones de AL estaban en inversión de portafolio (presentan mayor volatilidad).
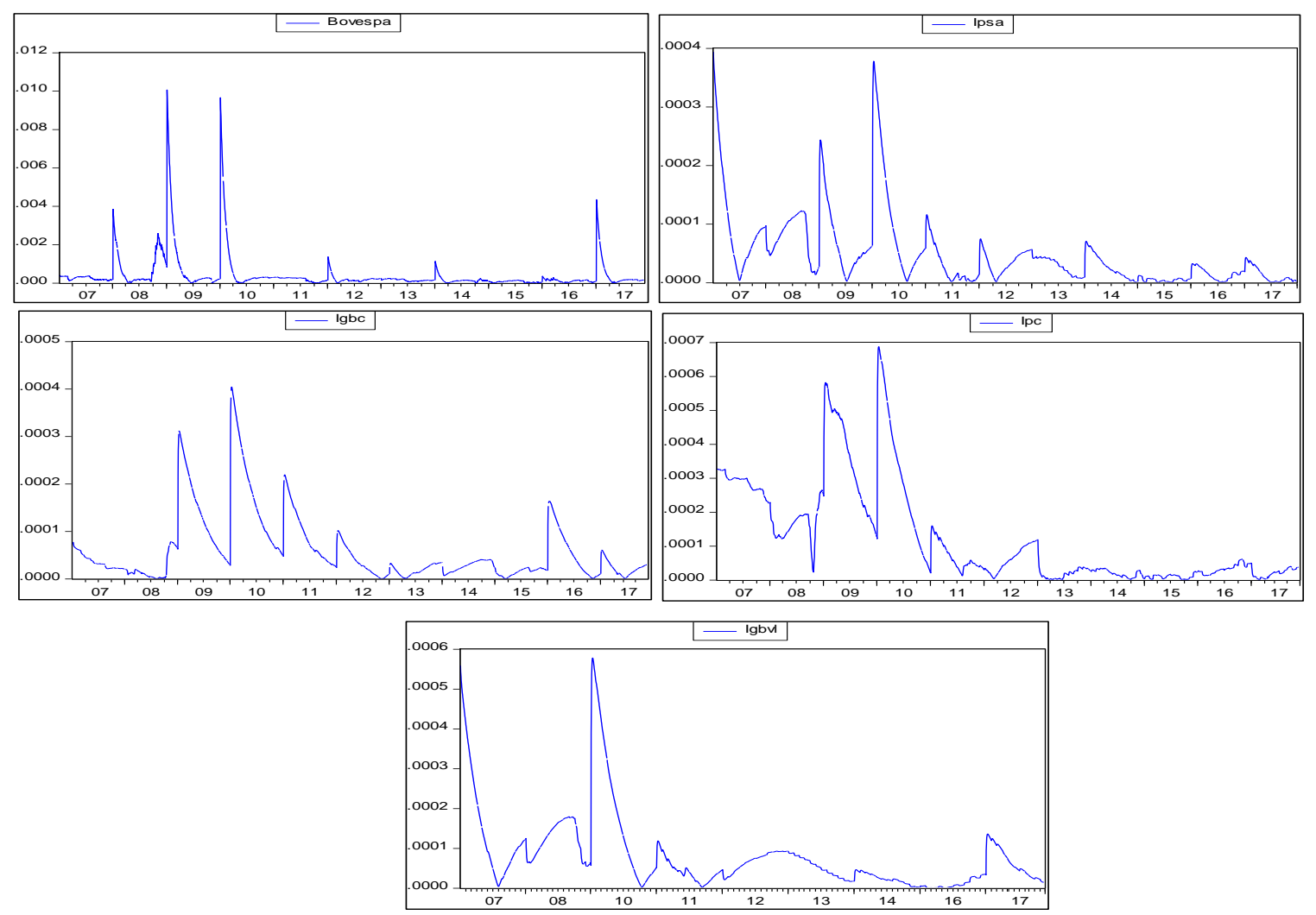

Figura 2. Volatilidad de los Índices Bursátiles por país.

Fuente: elaboración propia con datos de los Bancos Centrales (tomados en 2018). 


\section{Tabla 3}

Incidencia de la PMNC en la volatilidad de la bolsa

Variable dependiente: Volatilidad del índice bursátil

Método: Mínimos Cuadrados Ordinarios

Errores estándar ( )

\begin{tabular}{llllll}
\hline \multicolumn{1}{c}{ Var. Ind } & \multicolumn{1}{c}{ BOVESPA } & \multicolumn{1}{c}{ IPSA } & \multicolumn{1}{c}{ IGBC } & \multicolumn{1}{c}{ IPC } & \multicolumn{1}{c}{ IGBVL } \\
\hline Base Monetaria de EE.UU & $-3.4 \mathrm{E}-04 * * *$ & $5.06 \mathrm{E}-05^{* * *}$ & $8.24 \mathrm{E}-06^{* * *}$ & $-8.50 \mathrm{E}-05^{* * * *}$ & $8.30 \mathrm{E}-05^{* * *}$ \\
& $(3.12 \mathrm{E}-05)$ & $(1.37 \mathrm{E}-06)$ & $(1.19 \mathrm{E}-06)$ & $(2.397 \mathrm{E}-06)$ & $(2.51 \mathrm{E}-06)$ \\
Constante & $1.63 \mathrm{E}-03^{* * *}$ & $4.28 \mathrm{E}-04 * * *$ & $3.28 \mathrm{E}-04 * * *$ & $5.00 \mathrm{E}-04 * * *$ & $6.64 \mathrm{E}-04 * * *$ \\
& $(9.694 \mathrm{E}-05)$ & $(4.3 \mathrm{bE}-06)$ & $(3.78 \mathrm{E}-06)$ & $(9.54 \mathrm{E}-06)$ & $(8.90 \mathrm{E}-07)$ \\
Observaciones & 2697 & 2741 & 2658 & 2743 & 2232 \\
\hline
\end{tabular}

*** $\mathrm{p}<0.01,{ }^{* *} \mathrm{p}<0.05, * \mathrm{p}<0.1$

Fuente: elaboración propia con datos de los Bancos Centrales y la Reserva Federal.

La Tabla 3 muestra la relación y significancia de la volatilidad de cada índice bursátil en función de los cambios de la base monetaria, para el caso del BOVESPA e IPC, son los dos IB que presentan un coeficiente negativo, dadas las condiciones económicas del país y la estabilidad de su mercado financiero; dicho de otra manera, dentro de las bolsas más grandes la volatilidad se redujo, debido a que los agentes del mercado consideran que sus activos no están demasiado expuestos a los choques externos, permitiéndoles diversificar su portafolio de inversiones, y controlando la incertidumbre, mientras que en bolsas pequeñas, factores como la dependencia de inversión extranjera es mayor. Con respecto del IPSA, IGBC e IGBVL, sus niveles de volatilidad son similares, con una respuesta positiva ante la PMNC.

Uno de los factores más expuestos ante una crisis es la tasa de cambio, pues la pérdida del valor de la moneda genera encarecimiento de la deuda externa y en el mediano plazo inconvenientes con la meta de inflación, razón por la cual es importante determinar cómo fueron los procesos de volatilidad y ajuste del tipo de cambio en las EE de la región ante los choques de las medidas no convencionales. La estimación diseñada se hace mediante un modelo GARCH, lo que permite analizar cómo son los procesos de volatilidad de las tasas de cambio nominal: real (BRA), peso chileno (CHL), peso colombiano (COL) y sol peruano (PER) en función al índice de la base monetaria de Estados Unidos. 

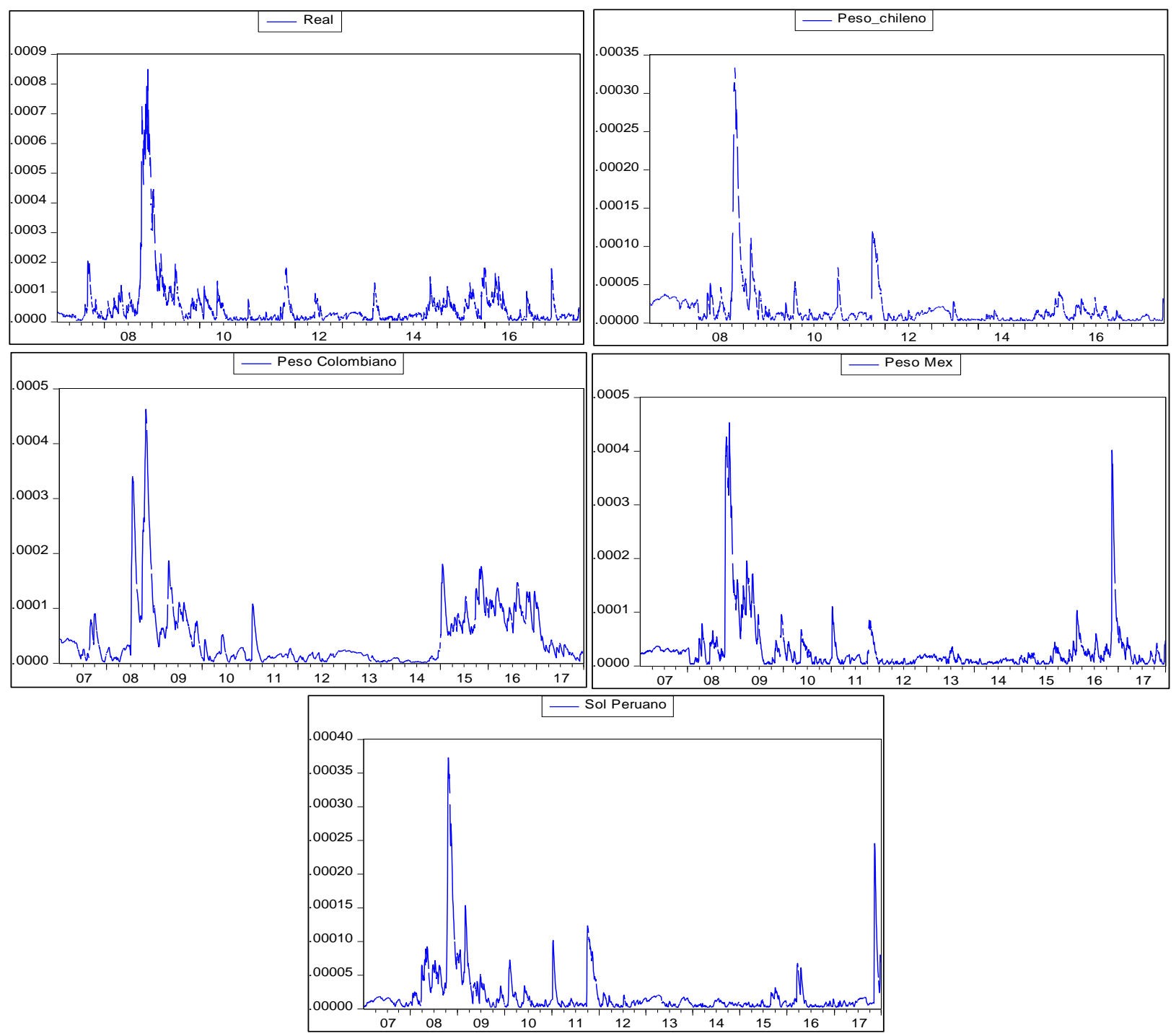

Figura 3. Volatilidad de las tasas de cambio.

Fuente: elaboración propia con datos de los Bancos Centrales (tomados en 2018).

Con respecto de los procesos de volatilidad, se destaca que la tasa de cambio (TC) de Colombia, fue uno de los más estables en el 2009, pues la exigencia de un nivel de encaje sobre la entrada de capitales controló la entrada de capitales más volátiles (Ocampo, 2009, p. 17). El deterioro de la balanza comercial de las EE de la región también ha afectado a las tasas de cambio, debido a que, desde inicio de la crisis, la tendencia de las TC ha sido clara, y es hacia la depreciación, efecto que es más notorio en el peso mexicano, seguido por el peso colombiano y sol peruano. De igual manera, entre el $2010 \mathrm{y}$ 2014, las TC no fueron tan volátiles en comparación a 2015-2017, ya que los flujos de capital en la 
región estaban apreciando las monedas de EE por sus rendimientos provenientes del sector de los commodities, y por la depreciación del dólar explicado por el momento de la economía mundial.

\section{Tabla 4}

Incidencia de la PMNC en la volatilidad de la tasa de cambio

Variable dependiente: Volatilidad del índice bursátil

Método: Mínimos Cuadrados Ordinarios

Errores estándar ( )

\begin{tabular}{|c|c|c|c|c|c|}
\hline Var. Ind & Real_BRA & Peso_CHL & Peso_COL & Peso_MEX & Sol_PER \\
\hline \multirow{2}{*}{ Base Monetaria de EE.UU } & $-1.27 \mathrm{E}-05^{* * *}$ & $-9.1 \mathrm{E}-06 * * *$ & $-7.97 \mathrm{E}-06 * * *$ & $-7.72 \mathrm{E}-06 * * *$ & $-7.27 \mathrm{E}-06^{* * *} *$ \\
\hline & $(1.35 \mathrm{E}-06)$ & $(6.6 \mathrm{E}-07)$ & $(9.80 \mathrm{E}-07)$ & $(8.71 \mathrm{E}-07)$ & $(5.57 \mathrm{E}-07)$ \\
\hline \multirow{2}{*}{ Constante } & $1.3 \mathrm{E}-04 * * *$ & $7.01 \mathrm{E}-05^{* * *}$ & $9.10 \mathrm{E}-05 * * *$ & $8.21 \mathrm{E}-05^{* * *}$ & $6.22 \mathrm{E}-05^{* * *}$ \\
\hline & $(4.32 \mathrm{E}-06)$ & $(2.1 \mathrm{E}-06)$ & $(3.13 \mathrm{E}-06)$ & $(2.87 \mathrm{E}-06)$ & $(1.78 \mathrm{E}-06)$ \\
\hline Observaciones & 2740 & 2740 & 2740 & 2740 & 2740 \\
\hline
\end{tabular}

$* * * \mathrm{p}<0.01, * * \mathrm{p}<0.05, * \mathrm{p}<0.1$

Fuente: elaboración propia con datos de los Bancos Centrales y la Reserva Federal.

La Tabla 4 muestra los resultados de la estimación de la volatilidad de las tasas de cambio en función de los cambios de la base monetaria, donde todos los resultados tienen significancia estadística. El modelo propuesto mostró que la mayor volatilidad aparece mientras ocurrió la expansión de la base monetaria en EE.UU, lo cual sugiere que la volatilidad de las tasas de cambio tiene sensibilidad a los cambios de la base monetaria; asimismo, dichos cambios sobre las TC afectaron a los flujos de capital, el nivel de inversiones y las expectativas de la tasa de interés de cada país.

Al definir el canal de transmisión de los efectos de las medidas no convencionales no tarda mucho en expandirse en una economía, debido a que las decisiones financieras toman menos tiempo en actuar que las tomadas por las autoridades monetarias. La causalidad encontrada es apoyada por la teoría, la cual sugiere que las mayores afectaciones y pérdidas que se han tenido para América Latina se da en sus bolsas de valores, dado que cuando estas se han desvalorizado los capitales migran del país, se 
reduce la inversión y la confianza de los agentes financieros, trayendo consigo que las empresas reporten pérdidas en sus dividendos, cancelación de operaciones y reducción de la capacidad productiva, esto en simultánea, obliga a los bancos centrales a modificar la tasa de interés e intervenir en el mercado cambiario para poder estabilizar la economía afectada por la incertidumbre y la volatilidad de los mercados accionarios.

En línea con la relación de causalidad expuesta, se construyen las funciones impulso-respuesta de Cholesky, que permiten ortogonalizar las perturbaciones mediante una descomposición triangular de la matriz de covarianzas, y se observa el comportamiento de las tasas de cambio ante los impulsos de los índices bursátiles, y estos últimos, de choques externos (Irastorza, Arce, y Arregui, 2010, p. 9). Un choque del BOVESPA al real brasileño hace que durante los tres primeros días de negociación este se deprecie para luego estabilizarse pasado el cuarto día desde el choque.

En relación a Chile se puede evidenciar que un choque por parte del IPSA genera una apreciación del peso chileno al día de recibir el choque, pero se estabiliza dos días después. En este caso, desde el inicio del choque hasta que se normaliza la tasa de cambio, transcurren cerca de cuatro días; la economía chilena tarda un día más en absorber el choque frente al caso de Brasil. En el caso del peso colombiano, se evidencia que ante choques al IGBC, durante el primer día, la tasa de cambio tiende a depreciarse y, en el tercer día, mantiene una tendencia para apreciarse con una duración de cuatro días. 

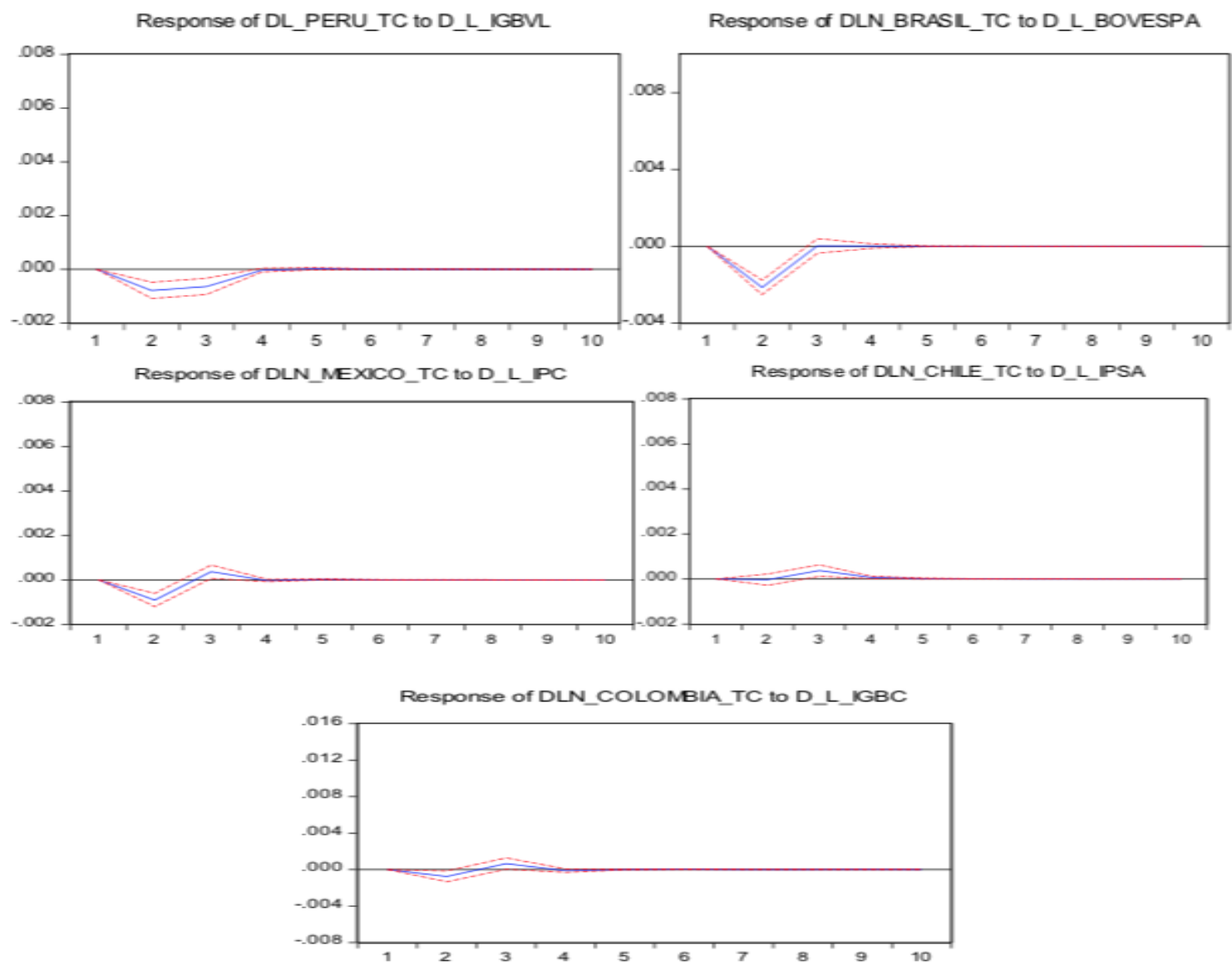

Figura 4. Funciones impulso-respuesta del TC de cada país a choques en IB.

Fuente: elaboración propia con Eviews 9.5.

Cuando se presenta un choque al IPC el primer efecto es la alta volatilidad que contiene este choque; esto genera una fuerte depreciación del peso durante los dos primeros días, pero un día antes de finalizar el choque, el peso se aprecia, no en la misma medida de la depreciación del primer día, y su duración es cuatro días también.

Por último, Perú, presenta una particularidad en materia de traspaso del choque. Cuando el IGBVL recibe un choque externo a diferencia de todas las otras monedas, el choque hace que el sol se aprecie a lo largo de los cuatro días que dura el efecto. Esto es explicado por dos razones claves: la primera, las medidas de regulación financiera que implementó Perú desde mediados de 2012 que buscaron que los flujos de capital con más alta volatilidad tuviesen impedimentos regulatorios para evitar que contagiaran la bolsa de valores de Lima. La segunda razón, es que el Banco Central del Perú tiene una política cambiaria poco flexible, que hace que participe de manera activa en las operaciones 
cambiarias diarias, además de tener en marcha todos los mecanismos de intervención, lo cual implica que, ante un choque externo de volatilidad e incertidumbre, los mecanismos mitiguen en gran parte las consecuencias internas al país.

En resumen, los choques de PMNC por parte de Estados Unidos determinaron el comportamiento cíclico de los mercados de activos financieros de la región desde el 2007 hasta 2017, pero dependió del país el nivel de la volatilidad. En definitiva, un choque externo demora cuatro días en ser absorbido y ajustado por la TC.

\section{Flexibilidad cuantitativa en EE.UU y flujos de capital hacia EE de AL}

Los flujos de capital en AL han estado sujetos a las dinámicas cíclicas de las economías desarrolladas, situación evidente en el comportamiento de la actividad bursátil de la región, y en el momento que detonó la crisis, los flujos en inversión de cartera mostraron una tendencia a la contracción hacia las EE, efecto que se corrigió en la región después de 2010, motivada por las bajas tasas de interés en las economías desarrolladas y la dinámica de las materias primas en la región.

Para explicar el impacto de las medidas no convencionales en los flujos de capital, es importante definir lo que determina la dinámica del comportamiento de estos flujos. En razón a esto, se estudian los flujos de inversión de cartera, específicamente las variaciones de los pasivos y activos externos, dado que es la entrada neta de capital extranjero en América Latina. Esta cartera comprende los instrumentos financieros en forma de títulos de participación y los títulos negociados en los mercados financieros externos.

Es importante destacar que los activos de cartera hasta el 2015 se contrajeron en la región, ya que la inestabilidad y riesgo en las economías desarrolladas era alto, generando un aumento de los flujos de inversión de cartera en la región explicada por la consolidación de las economías emergentes con el auge en los precios de las materias primas.

Los resultados evidencian que cuando la base monetaria se duplica la inversión de cartera del capital extranjero en el país aumenta un $0.71 \%$ con respecto de la dinámica económica del país (como proporción del PIB). En ese sentido, como el índice de la hoja de balance pasó de tener un valor de 1 
en noviembre de 2008 a cerca de 4 después de 2014 cuando finalizó la flexibilidad cuantitativa, entonces el efecto de la PMNC sobre el flujo de pasivos externos de cartera fue de $2.84 \%$ respecto del PIB.

\section{Tabla 5}

Impacto de las PMNC en la inversión de cartera de extranjeros

\begin{tabular}{|c|c|c|c|}
\hline & $\begin{array}{c}1) \\
\text { modelo_MCO }\end{array}$ & $\begin{array}{c}(2) \\
\text { modelo_FIXED }\end{array}$ & $\begin{array}{c}\text { (3) } \\
\text { modelo_RANDOM }\end{array}$ \\
\hline VARIABLES & IcarterapasivosPIB & IcarterapasivosPIB & IcarterapasivosPIB \\
\hline \multirow[t]{2}{*}{ Base Monetaria EE.UU } & 0.00192 & $0.00714 * * *$ & 0.00192 \\
\hline & $(0.00138)$ & $(0.00221)$ & $(0.00138)$ \\
\hline \multirow[t]{2}{*}{ Activos de Reserva_PIB } & $-0.245^{* * *}$ & $-0.318 * * *$ & $-0.245^{* * *}$ \\
\hline & $(0.0550)$ & $(0.0545)$ & $(0.0550)$ \\
\hline \multirow[t]{2}{*}{ Crecimiento económico } & 0.00359 & 0.00475 & 0.00359 \\
\hline & $(0.00405)$ & $(0.00389)$ & $(0.00405)$ \\
\hline \multirow[t]{2}{*}{ Tasa de Política } & $-0.243^{* * *}$ & $-0.213^{* *}$ & $-0.243^{* * *}$ \\
\hline & $(0.0512)$ & $(0.0903)$ & $(0.0512)$ \\
\hline \multirow[t]{2}{*}{ Cuenta corriente_PIB } & -0.000741 & $-0.00206^{* *}$ & -0.000741 \\
\hline & $(0.000813)$ & $(0.000830)$ & $(0.000813)$ \\
\hline \multirow[t]{2}{*}{ Crédito_PIB } & 0.00757 & $-0.0783 * * *$ & 0.00757 \\
\hline & $(0.00497)$ & $(0.0285)$ & $(0.00497)$ \\
\hline \multirow[t]{2}{*}{ Tasa de cambio } & $0.0623 * * *$ & 0.0347 & $0.0623 * * *$ \\
\hline & $(0.0219)$ & $(0.0638)$ & $(0.0219)$ \\
\hline \multirow[t]{2}{*}{ Constante } & $0.0160 * *$ & $0.0486^{* * *}$ & $0.0160 * *$ \\
\hline & $(0.00648)$ & $(0.0129)$ & $(0.00648)$ \\
\hline Observaciones & 208 & 208 & 208 \\
\hline R-cuadrado & 0.256 & 0.246 & \\
\hline Número de países & 5 & 5 & 5 \\
\hline
\end{tabular}

*** $\mathrm{p}<0.01, * * \mathrm{p}<0.05,{ }^{*} \mathrm{p}<0.1$

Fuente: elaboración propia en Stata 14 con datos de los Bancos Centrales y la Reserva Federal. 
La estimación de los determinantes de la inversión extranjera de cartera muestra que existe relación positiva con los cambios en la base monetaria de la Fed, efecto que se explica con el aumento considerable en los flujos hacia la región. Este aumento de inversión de cartera desde 2012 se debió al dinamismo y solidez financiera de los mercados de la región, acompañado del contexto internacional de bajos rendimientos, que aumentó la inversión en los mercados emergentes, en especial en AL (CEPAL, 2011). Dicha situación llevó a que la volatilidad de estos activos afectará de manera considerable los IB. Los países que más concentran inversión en cartera en su orden son: Brasil, México y Colombia.

Por medio del análisis de datos panel, se identifica cómo las decisiones de la Fed aumentaron la entrada de los flujos de capital, en especial después de 2012, cuando la región estaba en el auge de los commodities, ciclo que aumentó la participación de capitales extranjeros y ayudó a dinamizar los mercados financieros (Blancas, 2015). Expuesto de esta manera, con la normalización de las medidas no convencionales de la Fed, y la especulación que generó los cambios en los parámetros de política monetaria, los agentes financieros se enfocaran a invertir en las EE, en especial en activos financieros de alta rentabilidad y fáciles de comercializar, generando que la inversión de cartera fuera más volátil ante las decisiones de los inversores, y por consiguiente de la política monetaria.

Uno de los instrumentos más importantes que utilizó la Fed para reactivar la economía fue la hoja de balance, y el aumento excesivo del tamaño de esta generó especulación en los mercados financieros, ya que con el aumento de los pasivos de su balance se absorbió en una importante proporción con la compra de activos financieros, como hipotecas y bonos, limitando los activos financieros estables en su mercados financieros, llevando a la movilización de capitales hacia otros mercados estables a nivel mundial, en especial a las EE de América Latina. También, es clave destacar que con el QE3 el mercado financiero recibió un nivel extra de liquidez, aumentando la inversión en portafolio después de 2012.

Se debe hacer una aclaración con respecto de la situación anterior, ya que puede ser consideraba como contradictoria, pero no lo es, puesto que la Fed al reducir los activos financieros del mercado limitó las opciones para los inversionistas, muchos de ellos, estaban cerca de entrar en insolvencia, efecto que se marcó aún más cuando los activos sin ningún respaldo financiero se propagaron por la mayoría 
del mercado mundial, convirtiéndose en un problema de liquidez. En los dos escenarios la especulación fue elevada y el riesgo de invertir en Estados Unidos aumentaba, dejando como una alternativa invertir en otros mercados. Es así que la variación en los activos de reserva, la cuenta corriente, el crédito del sistema financiero y la tasa de cambio explican la tendencia de la ICP en la región.

La balanza de pagos de AL se vio fuertemente afectada por la PMNC, en relación a la volatilidad de los flujos de cartera, para la CEPAL (2018) la composición financiera de la balanza de pagos se ha concentrado en los flujos de portafolio; en especial los bonos que son mayores a los préstamos bancarios, efecto derivado del aumento de los pasivos de la hoja de balance de la Fed, que generó la recomposición por los bonos con mayor nivel de volatilidad. La balanza de pagos de AL como se ha venido mostrando, fue una de las más expuestas a los choques externos. En relación, a la exposición del sector financiero principalmente a los choques externos, las diferentes autoridades monetarias de la región han tomado medidas macroprudenciales para estabilizar la economía y controlar la especulación en el sistema financiero.

\section{Tabla 6}

Impacto de las PMNC en la inversión en activos externos de cartera por nacionales

\begin{tabular}{|c|c|c|c|}
\hline & $\begin{array}{c}\text { (1) } \\
\text { modelo_MCO }\end{array}$ & $\begin{array}{c}\text { (2) } \\
\text { modelo_FIXED }\end{array}$ & $\begin{array}{c}\text { (3) } \\
\text { modelo_RANDOM }\end{array}$ \\
\hline VARIABLES & I_carteraactivosPIB & I_carteraactivosPIB & I_carteraactivosPIB \\
\hline \multirow[t]{2}{*}{ Base Monetaria EE.UU } & $0.00360 * *$ & $-0.00620 * *$ & $0.00360 * *$ \\
\hline & $(0.00160)$ & $(0.00243)$ & $(0.00160)$ \\
\hline \multirow[t]{2}{*}{ Cuenta corriente_PIB } & $-0.00345 * * *$ & $-0.00327 * * *$ & $-0.00345 * * *$ \\
\hline & $(0.000920)$ & $(0.000954)$ & $(0.000920)$ \\
\hline \multirow[t]{2}{*}{ CréditoPIB } & $-0.0363 * * *$ & $0.127 * * *$ & $-0.0363 * * *$ \\
\hline & $(0.00608)$ & $(0.0341)$ & $(0.00608)$ \\
\hline \multirow[t]{2}{*}{ Crecimiento económico } & $0.0153 * * *$ & $0.0176^{* * *}$ & $0.0153 * * *$ \\
\hline & $(0.00513)$ & $(0.00472)$ & $(0.00513)$ \\
\hline Constante & -0.00697 & $-0.0723 * * *$ & -0.00697 \\
\hline
\end{tabular}


$(0.00556)$

Observaciones

R-cuadrado

Número de países
210

0.238
5
$(0.0144)$

210

0.198

$(0.00556)$

210

5

$* * * \mathrm{p}<0.01, * * \mathrm{p}<0.05, * \mathrm{p}<0.1$

Fuente: elaboración propia en Stata 14 con datos de los bancos centrales.

En la estimación, ICA responde de manera inversa ante los cambios de la base monetaria, debido a que cuando aumenta la liquidez en los mercados financieros desarrollados los mercados lo ven como una señal de contracción de dichas economías, generando el aumento del riesgo e incertidumbre en las economías desarrolladas. Esto motiva a que la inversión de cartera de activos externos de cada país se vea afectada por factores como la volatilidad y el contagio financiero masivo.

La tendencia de contracción de los activos externos de cartera es explicada en el informe de las Naciones Unidas (UNCTAD, 2017), que señala cómo después de 2015 la contracción de dichos flujos en las EE aumentó por factores como riesgos políticos, exceso de endeudamiento y fuertes presiones a la devaluación. Para De la Torre et al. (2014) la contracción de inversión en el extranjero está explicada, no sólo por la normalización monetaria de la Fed, sino también por el incremento de los costos de capital para las EE, originada porque los bonos del Tesoro estadounidense aumentaron su rentabilidad, generando que varias economías de la región incrementarán la tasa de política monetaria o permitieran la depreciación de la moneda para mitigar el choque externo.

La reducción de ICA está asociada con la contracción de la participación en títulos de otros países, ya que después de 2015, la posición de inversión de varias economías en la región cambió reduciendo los activos de inversión, fenómeno agudizado con el encarecimiento de la deuda externa y el deterioro de la cuenta corriente, pues es una señal para los inversionistas de un mayor nivel de riesgo e inestabilidad económica del país. 


\section{CONCLUSIONES}

El objetivo principal del presente trabajo es determinar los efectos de la PMNC de Estados Unidos sobre los mercados de las EE en AL, escenario que se logró mostrar por medio de dos estimaciones, una es la volatilidad que registraron los IB y la TC de las EE de la región ante los cambios de política monetaria de Estados Unidos en el marco de la crisis. Para el caso los índices bursátiles, a pesar de registrar fuertes pérdidas a lo largo de 2007-2017, se lograron mantener estables en los años posteriores, pero frente a las tasas de cambio, y según el tiempo promedio que tarda cada economía en controlar un choque externo fueron los que más consecuencias y costos le generaron a la región, pues al tener depreciaciones de hasta un $40 \%$ con respecto del dólar, era indudable los efectos negativos hacía cada economía, efecto que respalda los resultados obtenidos de la estimación de los flujos de capital en función de los cambios de la base monetaria.

De igual manera, los flujos de capital más expuestos a los choques externos son la inversión de cartera hacia $\mathrm{AL}$, que en parte cambió por las variaciones en la base monetaria de EE.UU; y al igual que en los resultados de Chen et al. (2014) y Bowman et al. (2015), las características propias de los países son relevantes para identificar la relación señalada. Los activos financieros contabilizados en los flujos de cartera tienen menor maduración, un riesgo más alto y periodos de vencimiento a corto plazo, concluyendo así que el cambio en la hoja de balance de la Fed tuvo un mayor impacto en la inversión de cartera que en la inversión extranjera directa (se realizó ejercicios econométricos con la inversión extranjera directa y se evidenció que no existen efectos estadísticamente significativos).

El rendimiento de los indicadores bursátiles tiene relación positiva con la tendencia de la inversión de cartera, lo que plantea algunos escenarios futuros en las EE de América Latina. En el momento en que los precios de los activos financieros disminuyan por la reducción de liquidez a nivel mundial, durante el proceso de reajuste de la hoja de balance, generará especulación en los mercados, que podría resultar en una fuga de capitales motivadas por la continua depreciación de las monedas, e incremento de las tasas de interés, dejando expuesta a la balanza de pagos y el encarecimiento de la deuda externa.

Una de las hipótesis planteadas para este trabajo es que la PMNC afectó de manera negativa a los flujos de capital, situación que se debe aclarar; en un primer momento la PMNC motivó la salida de capitales de Estados Unidos hacia otras economías en búsqueda de rentabilidad, situación clara en AL, 
en especial con el auge de las materias primas, las mayores entradas de flujos de cartera en las economías emergentes. En un segundo momento del tiempo, la recuperación de las economías desarrolladas condujo a una reversión de los flujos de capital, sin considerar la caída de los precios de los commodities y la contracción de algunas economías asiáticas, que acentuaron la salida de capitales de la región, no sólo los flujos salían de la región por la recuperación de las economías desarrollados, sino también porque la región se empezaba a desacelerar por los factores anteriormente mencionados.

Por último, aunque las $\mathrm{EE}$ de $\mathrm{AL}$ analizadas presentaron un comportamiento estable, las más vulnerables en relación a la actividad bursátil fueron México, Colombia y Brasil, que reportaron mayores niveles de volatilidad en el comportamiento de sus índices bursátiles; en parte, se puede considerar que la variabilidad de estos índices se debió a la dependencia con el sector de mineroenergético y, al mismo tiempo después de 2015 se contrajo el ingreso de flujos de capital en América Latina. La agenda de investigación queda abierta para abordar el papel de la PMNC en relocalización de la inversión extranjera en los diferentes sectores de AL, y así observar los efectos de largo plazo.

\section{REFERENCIAS}

(1) Ahmed, S., y Zlate, A. (2014). Capital flows to emerging market economies: a brave new world? Journal of International Money and Finance, 48, 221-248.

(2) Blancas, A. (2015). Fuga de Capitales en México: análisis y propuesta de medición. Revista Problemas del Desarrollo-UNAM, 24-26.

(3) Bowman, D., Londono, J., y Sapriza, H. (2015). US unconventional monetary policy and transmission to emerging market economies. Journal of International Money and Finance, 55, 27-59.

(4) Calvo, G., y Perry, G. (Marzo de 2015). El Fin de un Cliclo en América Latina y sus Riesgos. Comité Latino Maericano de Asuntos Financieros. Obtenido de http://vox.lacea.org/?q=blog/ciclo-latam-declaracion-claaf

(5) Caruana, J. (2011). El papel de los Bancos Centrales después de la crisis. Bank for Internacional Settlements- Acto Academico OBCE.

(6) CEPAL. (2011). Estudio Económico de América Latina y el Caribe. Publicación de las Naciones Unidas.

(7) CEPAL. (2016). Panorama Social de América Latina 2016. Obtenido de https://www.cepal.org/es/publicaciones/41598-panorama-social-america-latina-2016

(8) CEPAL. (2018). Balance Preliminar de las Economías de América Latina y el Caribe. Comisón Economica para América Latina y el Caribe, 33-38. 
(9) Cepalstat. (octubre de 2018). www.interwp.cepal.org. $\quad$ Obtenido http://interwp.cepal.org/sisgen/SisGen_MuestraFicha.asp?indicador=2207yid_estudio=131

(10) Cepalstat. (octubre de 2018). www.interwp.cepal.org. Obtenido de http://interwp.cepal.org/sisgen/SisGen_MuestraFicha.asp?indicador=2767yid_estudio=812.

(11) Chen, M., Griffoli, M., y Sahay, M. (2014). Spillovers from United States monetary policy on emerging markets: different this time? (No. 14-240). International Monetary Fund.

(12) Dahlhaus, T., y Vasishtha, G. (2014). The impact of US monetary policy normalization on capital flows to emergingmarket economies (No. 2014-53). Bank of Canada Working Paper.

(13) De la Torre, A., Beylis, G., y De Piniés, J. (2014). LAC Semiannual Report April 2014: International Flows to Latin America-Rocking the Boat? The World Bank.

(14) Gilchrist, S. (2014). The Response of Sovereign Bond yields to U.S Monetary Policy. Jacques Polak Annual Research Conference.

(15) Hausman, J. (1978). Specification tests in econometrics. Econometrica: Journal of the econometric society.

(16) Irastorza, E., Ullibarri, M., y Arregui, Z. (2010). Un modelo VAR aplicado al empleo y las horas de trabajo. Estadística Española.

(17) Labra, R., y Torrecillas, C. (2014). Guía CERO para datos de panel. Un enfoque práctico. UAM-Accenture Working Papers.

(18) Martin, A., García, V., y Arilla, M. (2010). Crisis Económicas a lo Largo de la Historia . Cuadernos de Formación. Colaboración.

(19) Monsegny, M., y Cuervo, E. (2008). Modelos Arch, garch y EGarch: aplicaciones a series financieras. Cuadernos de Economía, v XXVII, 16-18.

(20) Morales, P. (2014). La eficacia de las políticas monetarias de Quantitative Easing llevadas a cabo por la reserva federal de los Estados Unidos y por el Banco Central Europeo. Universidad Pontificia Comillas ICAI-ICADE. Madrid. Obtenido de https://repositorio.comillas.edu/rest/bitstreams/1087/retrieve

(21) Ocampo, J. (2009). Impactos de la crisis financiera mundial sobre América Latina. Revista cepal .

(22) Ocampo, J. (2009). La crisis económica global: impactos e implicaciones para América Latina. Nueva Sociedad 224, 67.

(23) Orgaz, L., y Carrasco, M. (2011). El creciente peso de las economías emergentes en la economía y gobernanza mundiales: los países BRIC. 52.

(24) Otero, D. (2015). Impacts of monetary policy and transmission channel in Latin-American countries with an inflation targeting scheme. Ensayos sobre POLÍTICA ECONÓMICA 33.SPE76, 75.

(25) Perazzi, J., y Merli, G. (2013). Modelos de regresión de datos panel y su aplicación en la evaluación de impactos de programas sociales. Telos: Revista de Estudios Interdisciplinarios en Ciencias Sociales, 130.

(26) Ramírez, A. y Rodríguez, H. (2013). Un análisis VAR estructural de política monetaria en Colombia. Revista de la Facultad de Ciencias Económicas: Investigación y Reflexión, XXI (2),17-41. Obtenido de https://www.redalyc.org/pdf/909/90930501003.pdf

(27) Ricketts, L. (2011). Quantitative Easing Explaines. Federal Reserve bank of St Louis, 2-8. 
(28) Rincón, H. (2013). Flujos de capitales, choques externos y respuestas de política en países emergentes. Obtenido de http://www. banrep. gov. co/libro-flujos-capitales

(29) Rubai, G. (2013). La Generalización del Quantitative Easing y sus repercusiones en la Estabilidad Financiera Global. Instituto Español de Estudios Estrategicos.

(30) Tillmann, P. (2016). Unconventional monetary policy and the spillovers to emerging markets. Journal of International Money and Finance, 66, 136-156.

(31) Toro, J., Mora, R., y Parra, D. (2012). Flujos de capital, la crisis financiera internacional y los desbalances macroeconómicos. Borradores de Economía; No. 720.

(32) Wooldridge, J. (2010). Introduccion a la economía, un enfoque moderno. (4 ${ }^{a}$. Edición). Mexico. Obtenido de https://www.academia.edu/30200962/Introducción_A_La_Econometría_4edi_Wooldridge 